\title{
Relationships Among Retinal Nerve Fiber Layer Thickness, Vascular Endothelial Growth Factor, and Cognitive Impairment in Patients with Schizophrenia
}

\author{
Yanhong Liu ${ }^{1,2}$ \\ Jingxu Chen ${ }^{1,2}$ \\ Lvzhen Huang ${ }^{3}$ \\ Shaoxiao Yan ${ }^{1,2}$ \\ Qingtao Bian ${ }^{1,2}$ \\ Fude Yang ${ }^{1,2}$ \\ 'Huilongguan Clinical Medical School, \\ Peking University, Beijing, People's \\ Republic of China; ${ }^{2}$ Beijing Huilongguan \\ Hospital, Beijing, People's Republic of \\ China; ${ }^{3}$ Ophthalmology Department, \\ People's Hospital of Peking University, \\ Beijing, People's Republic of China
}

Correspondence: Fude Yang Huilongguan Clinical Medical School, Peking University, Nandian, Road,

Changping District, Beijing, 100096,

People's Republic of China

Tel +86-|0-8302425|

Fax +86-10-83024302

Email yangfd2021@I63.com
Background: Studies have suggested retinal nerve fiber layer (RNFL) involvement in the pathogenesis of schizophrenia. Additionally, research has shown that vascular endothelial growth factor (VEGF) potentially contributes to the pathophysiology of psychiatric disorders. Therefore, this study aimed to investigate VEGF, RNFL, and correlations with cognitive impairments in schizophrenia patients.

Methods: Patients with schizophrenia $(\mathrm{n}=138)$ were compared to healthy controls $(\mathrm{n}=$ 160). RNFLs were measured with optical coherence tomography (OCT). The Stroop color and word test (SCWT) was used to evaluate neurocognition. Blood samples were collected to measure VEGF. SPSS 20.0 was used to perform analysis of covariance, t-tests, partial correlation analysis, and linear regression.

Results: Thinner RNFLs were found in schizophrenia patients $(p<0.001)$. RNFL showed a significant correlation with SCWT scores (all $p<0.05$ ). Serum level of VEGF was lower in patients with schizophrenia $(p=0.010)$. Total and inferior RNFL thicknesses of right eyes were positively correlated to VEGF level (RNFL total thickness $p=0.032$, inferior thickness $p=0.014)$. Total RNFL thicknesses were shown to be reduced following a prolonged duration of illness (both $\mathrm{p}<0.01$ ).

Conclusion: These findings suggest that patients with schizophrenia have degeneration with RNFL thickness following illness duration, which may contribute to neurocognitive impairments observed in schizophrenia. VEGF is speculated to play some important role on RNFL degeneration with schizophrenia patients.

Keywords: RNFL, VEGF, cognitive impairment, schizophrenia

\section{Introduction}

Neurodegeneration of the brain may pertain to a variety of functional deficits that are essential features of the schizophrenia. ${ }^{1}$ The visual nervous system in the brain primarily originates from the neuroectoderm. Retinal anomalies also present in schizophrenia patients, ${ }^{2}$ including atrophy of retinal nerve fiber layers (RNFL). ${ }^{3-5}$ The thickness decrease observed in the RNFL could reflect retinal cell loss. ${ }^{6}$

Studies have shown both structural and functional deficiency in the visual afferent pathways of patients with schizophrenia. ${ }^{7,8}$ For example, inherent connectivity of the primary afferent visual pathway has been found to be reduced in schizophrenia patients, and impaired visual object perception is considered 
a pathogenic risk factor of schizophrenia. ${ }^{9}$ Deviation in the process of visual information transmission is more common in schizophrenia patients. ${ }^{10}$ The retina as the starting station of the visual pathway through the brain is an especially accessible substrate for investigating the brain pathology of schizophrenia. ${ }^{11}$ In vivo visualization of the retina can be achieved by optical coherence tomography (OCT), a non-invasive, fast imaging technique. ${ }^{10}$

Serum vascular endothelial growth factor (VEGF) has been shown to provide protection in the inflammatory process of schizophrenia. ${ }^{12}$ Kurihara et al reveal an essential role of VEGF in maintaining choroid vasculature and cone photoreceptors, critical for central and color vision. ${ }^{13}$ However, the effect of VEGF on the RNFL of schizophrenia patients remains unknown.

The Stroop color-word test (SCWT) is widely utilized as a measurement of neurocognition, which is thought to reflect frontal lobe function. The Stroop paradigm requires an intact visual system, especially color vision and visual acuity. ${ }^{14}$ While neuropsychology textbooks warn that visual dysfunction may interfere with Stroop performance, ${ }^{15}$ how the retina affects the visual-related cognitive impairment observed in schizophrenia remains unknown.

We conducted this study to investigate structural changes in the retina of individuals with schizophrenia and its correlations with cognitive performance, as assessed by the SCWT. We predicted that patients with schizophrenia would show reduced RNFL thickness, and that would be associated with visual cognitive impairments under the imbalanced level of VEGF.

\section{Methods and Materials}

\section{Recruitment of Subjects}

This cross-sectional study enrolled patients with schizophrenia and healthy controls aged $18-60$ years. The present study was conducted from July 2018 to January 2020. Two experienced psychiatrists confirmed the diagnosis of schizophrenia based on the Structured Clinical Interview for the Diagnostic and Statistical Manual of Mental Disorders-IV (Structured clinical interview for diagnosisI version, SCID-I). All patients had been receiving stable doses of antipsychotic drugs for at least three months before entry into the study. We recorded smoking status as an interfering factor considering the influence of smoking on cognition and the retina. ${ }^{16,17}$ Healthy controls recruited from voluntary hospital staff were also evaluated by SCID-I to rule out Axis I disorders. All participants fulfilled the exclusion criteria and were free of central nervous system diseases, head trauma, hypertension, diabetes mellitus, drug/alcohol abuse or addiction, glaucoma, cataract, high astigmatism (sphere dpt [diopter] $>4$ and cylinder dpt $>2$ ), high myopia ( $>6.0$ diopters), visual field defect, optic media opacities, chromatodysopia, or other significant medical illnesses (eg, cancer, ongoing infection). The best corrected visual acuity and body mass index (BMI) were also recorded. Due to the particularity of the condition of schizophrenic patients, guardians need to supervise and drive on behalf of their behavioral ability. Especially during the development of acute symptoms. All subjects or guardians provided written informed consent. Our study complies with the Declaration of Helsinki. The project was approved by the Institutional Review Board of Beijing Huilongguan Hospital (number 2018-33). ${ }^{18}$

\section{Psychological Evaluation}

The SCWT was used to evaluate the word/color processing speed and visual cognitive flexibility in all subjects. ${ }^{19}$ The SCWT features a three-page test booklet. The first page consists of the words "red," "yellow," and "blue" printed in black ink; the second page consists of columns of circles printed in red, yellow, or blue ink; and the third page consists of the words "red," "yellow," and "blue" printed in red, yellow, or blue ink, but the words and printed colors never match. Subjects were required to read the words and/or name the color on each page. ${ }^{20}$ The SCWT includes four direct scores, based on the time of items correctly completed on each of the three stimulus sheets (word reading time [WRT], color naming time [CRT], color-word reading time [CWRT]; and word-color naming time, [WCRT] was recorded).

The Positive and Negative Syndrome Scale (PANSS) assessment was performed on subjects with schizophrenia. This scale was designed and standardized to assess the presence and severity of different types of schizophrenia symptoms.

The PANSS consists of 7 positive scale items, 7 negative scale items, and 16 general psychopathology scale items, resulting in 30 total items to assess symptoms of schizophrenia. Three additional supplementary items are added to assess the risk of aggression. This study was evaluated by two psychiatrists who were blind to the study design and grouping. The kappa value of the consistency test was $0.85 .^{21}$ 


\section{Measurement of RNFL with OCT}

The best corrected visual acuity of each eye was measured via Snellen visual acuity. An anterior slit lamp microscope and Goldman tonometer were used to exclude eye diseases and measure intraocular pressure. Ophthalmologists used a 90D focusing lens for fundus examination to observe and exclude diseases of the RNFL or the macula around the disc. OCT 2002 type (Topcon, Japan) was used to scan both eyes (see Figure 1). Optic data were obtained with an OCT raster centered tu the optic nerve head in the " $6 \mathrm{~mm}$ $\times 6 \mathrm{~mm}$ " cube with a $512 \times 218$ scan and 200 pixels. The macular region was scanned in a radial " $6 \mathrm{~mm} \times 6 \mathrm{~mm}$ " region at $1024 \times 12$ and 200 superpixels. OCT software (version 8.42) automatically focused on the center of the disc or macular region then extracted a circle with a diameter of 3-4 $\mathrm{mm}$ to measure the RNFL thickness. RNFL thickness was measured at the 12 clock positions and in the four $90^{\circ}$ quadrants of the whole eyeball, including the superior, inferior, nasal and temporal quadrants. All data of retina were all automatically calculated by OCT All the above ophthalmic methods and data were recorded as described in our earlier studies. ${ }^{18}$

\section{Blood Sample Tests}

Serum samples were collected by nurses following fast $(6: 30 \mathrm{am})$. Blood was naturally coagulated at room temperature for 10-20 minutes, centrifuged for approximately 10 minutes (3000 rpm), and then the serum was separated, rapidly frozen, and stored at $-80^{\circ} \mathrm{C}$ until analysis.VEGF levels in serum were determined with enzyme-linked immunosorbent assay (ELISA) kits (Abcam, USA) by two technicians who were blinded to the status of the subjects. The absorbance (OD value) was measured at $450 \mathrm{~nm}$ by a microplate reader, and the concentration of VEGF was calculated by a standard curve. Interassay variances were below $8 \%$. The detection limit of the assay was $9 \mathrm{pg} / \mathrm{mL}$. Additionally, each serum sample was assayed in duplicate for glucose, triglyceride, and cholesterol, as reported earlier. ${ }^{18}$

\section{Statistical Analysis}

Data were analyzed with SPSS 20.0 software. A ShapiroWilk test was used to verify whether the data followed a normal distribution. Independent t-tests and Chi-square tests were used to compare the demographic and clinical data between patients and normal controls. Analysis of covariance (ANCOVA) was also used to compare the two groups, after controlling for age, sex, and smoking. Partial correlation analysis was used to assess the correlations between RNFL thickness and SCWT of the participants, controlling for age, sex, and smoking. Multiple linear regression analysis was used only in the schizophrenia patients. Age of onset, VEGF and duration of illness were set as independent variables and RNFL thickness of both eyes was set as dependent variables, after controlling for sex education and smoking. Total RNFL thickness, cup volume and average macular thickness were set as independent variables, scores of PANSS and Stroop test were

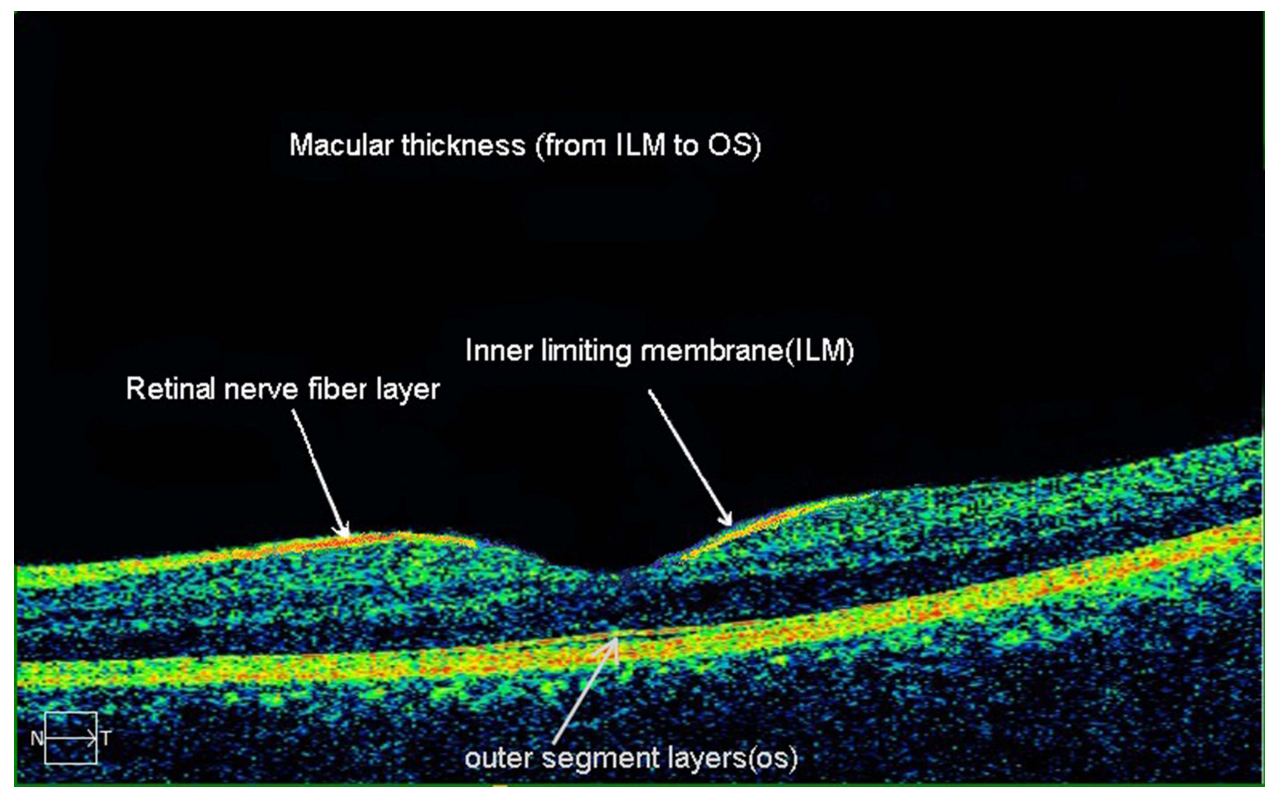

Figure I Retinal nerve fiber layer of OCT. 
set as the dependent variable, after controlling for age, sex and smoking. A $p$-value $<0.05$ for a two-tailed test was considered statistically significant.

\section{Results}

A total of 138 patients with schizophrenia and 160 healthy controls were enrolled in our study. As shown in Table 1, we found significant differences in age $(p=0.007)$, sex ( $p=0.001)$, and smoking condition $(p<0.001)$ between the patients and normal controls, while there were no significant differences in BMI or years of education between the two groups. The level of VEGF was lower in patients with schizophrenia $(p=0.010)$. Neurocognition, as assessed via the SCWT, was significantly impaired in the schizophrenia group (all $p<0.05$ ). The schizophrenia group showed significant delay in color naming time, color word reading time and word color naming time of the SCWT when compared to healthy controls (all $p<0.001$ ).

As shown in Table 2, the RNFL thicknesses of both eyes in schizophrenia patients were thinner than that observed in healthy controls $(p<0.001)$. By analyzing the relationships between color perception scores and retinal structure in the whole subject, we found slight correlations between the total RNFL and CWRT (right eye: $p=0.018, r=-0.192$; left eye: $p<0.001, \mathrm{r}=-0.341)$ as well as between the superior and inferior RNFL part of the SCWT results (Figure 2). In Table 3, cognitive testing scores of schizophrenia patients showed no significant correlations with the total of RNFL thicknesses except the CWRT scores $(\mathrm{P}=0.010)$. There were no significant relationships between cognitive testing scores and the cup volume or average macular thickness (all $\mathrm{p}>$ $0.05)$. Only negative symptoms of PANSS showed minor correlations with total RNFL thicknesses $(p=0.050)$.

In patients with schizophrenia, the total and inferior RNFL thicknesses of right eyes were shown to be thinner following the decreased VEGF level in schizophrenia (RNFL total thickness $p=0.032$ and inferior thickness $p=0.014$; see Table 4). Age of onset showed no relationships with all RNFL thickness (all $p>0.05$ ). Interestingly, total RNFL thickness was shown to be reduced following a prolonged duration of illness (right eye: $\beta=-0.992, \mathrm{~F}=-2.708, p=0.009$; left eye: $\beta=-0.496$, $\mathrm{F}=-2.980, p=0.004)$; a result also observed in the superior RNFL (right eye: $\beta=-1.334, \mathrm{~F}=-2.665, p=0.010$; left eye: $\beta$ $=-0.627, \mathrm{~F}=-1.676, p=0.099)$. However, the inferior RNFL showed no relationship with duration of illness $(p>0.05)$.

\section{Discussion}

As we know, this is the first study for correlations among retinal nerve fiber layer thickness, vascular endothelial growth factor, and cognitive impairment in patients with schizophrenia in Asia. Our cross-sectional study revealed that, compared with healthy controls, RNFL thickness in individuals with schizophrenia exhibits thinner, which may have some relationship with visual cognitive impairment. Furthermore, there appeared to be a relationship between illness duration and total RNFL thickness, but not inferior RNFL thickness. VEGF may play an important role on RNFL degeneration with schizophrenia patients.

RNFL and macular thickness were shown to be thinner in both eyes of patients with schizophrenia, which is consistent with observations in previous research. ${ }^{22-24}$ Moreover, following a prolonged illness duration, the RNFL thickness of the patients exhibited, decreased. Our results were consistent with a preclinical study on schizophrenia. ${ }^{25}$ All these studies provide evidence for the notion of structural and functional changes in retinal integrity of patients with schizophrenia, which maybe supports the neurodegenerative hypothesis of schizophrenia. $^{26,27}$

It should be noted that there appeared to be a relationship between illness duration and superior RNFL thickness. However, the same relationship was not observed for inferior RNFL thickness. Further, there were differences observed in associations between the left RNFL and Stroop results. The seemingly unbalanced bilateral development of the eyes observed in the current study may be related to the hypothesis of asymmetric brain development in schizophrenia. ${ }^{28,29}$

We did not find significant correlations between cup volume with cognitive impairment in schizophrenia. These results were not consistent with the results of a previous study, which showed increased cup volume and cup-todisc ratio in patients with schizophrenia who had comorbid diseases, and that the enlargement of cup volume was related to cognitive impairment. ${ }^{24}$ There was no apparent correlation between cup volume and cognitive symptoms in the absence of co-morbidity factors, like diabetes and hypertension, in our study. These different results may be due to different methods of measuring cognitive ability and differences in the samples studied.

A study reported a parallel alteration of VEGF serum and cerebrospinal fluid levels in subjects with neurotuberculosis. ${ }^{30}$ In our study, serum VEGF levels were lower in patients with schizophrenia than those 
Table I Participant Demographics and Differences in the SCWT Between Individuals with Schizophrenia and Healthy Controls

\begin{tabular}{|c|c|c|c|c|}
\hline Mean \pm SD & Schizophrenia Group $(n=\mid 38)$ & Control Group $(n=160)$ & $\mathrm{t} / \chi^{2}$ & $p$ \\
\hline Age, y & $45.09 \pm 12.47$ & $41.43 \pm 9.50$ & 2.737 & $0.007^{*}$ \\
\hline Age onset, y & $23.28 \pm 9.63$ & - & & \\
\hline Sex Female, $n$ & 66 & 106 & 33.470 & $0.001 *$ \\
\hline Male, $n$ & 72 & 54 & & \\
\hline Education, $y$ & $12.43 \pm 2.49$ & $12.70 \pm 2.78$ & 0.689 & 0.492 \\
\hline Duration of illness, $y$ & $21.74 \pm 13.51$ & - & & \\
\hline Smokers, n (\%) & $38(27.53)$ & $8(5.80)$ & 51.164 & $<0.00 I^{*}$ \\
\hline BMI $\left(\mathrm{kg} / \mathrm{m}^{2}\right)$ & $24.32 \pm 4.18$ & $23.40 \pm 3.16$ & 1.934 & 0.054 \\
\hline Eye pressure left $(\mathrm{mmHg})$ & $15.38 \pm 3.12$ & $15.35 \pm 3.04$ & 0.082 & 0.935 \\
\hline Right $(\mathrm{mmHg})$ & $15.29 \pm 3.43$ & $15.13 \pm 2.75$ & 0.395 & 0.693 \\
\hline Diopters (D) left eye & $3.10 \pm 1.71$ & $2.68 \pm 1.18$ & 0.856 & 0.396 \\
\hline Right eye & $2.86 \pm 1.34$ & $2.98 \pm 1.20$ & -0.269 & 0.789 \\
\hline $\mathrm{SBP}^{\prime}(\mathrm{mmHg})$ & $111.38 \pm 9.51$ & $110.02 \pm 8.96$ & 0.319 & 0.750 \\
\hline $\mathrm{DBP}^{2}(\mathrm{mmHg})$ & $70.91 \pm 5.64$ & $71.97 \pm 6.31$ & -1.475 & 0.141 \\
\hline Glucose $(\mathrm{mmol} / \mathrm{L})$ & $5.29 \pm 1.44$ & $5.39 \pm 1.49$ & -0.486 & 0.628 \\
\hline Cholesterol (mmol/L) & $4.20 \pm 0.96$ & $4.78 \pm 0.78$ & -0.097 & $<0.00 I^{*}$ \\
\hline Triglyceride (mmol/L) & $1.67 \pm 0.89$ & $1.46 \pm 0.99$ & 1.654 & 0.100 \\
\hline $\operatorname{VEGF}^{3}(\mathrm{pg} / \mathrm{mL})$ & $960.74 \pm 149.80$ & $1032.36 \pm 182.31$ & -2.597 & $0.008^{*}$ \\
\hline \multicolumn{5}{|l|}{ Stroop color word test } \\
\hline Word reading time (WRT, s) & $16.00 \pm 4.45$ & $13.82 \pm 9.52$ & 2.402 & 0.017 \\
\hline Color naming time (CRT, s) & $24.95 \pm 9.15$ & $16.04 \pm 3.67$ & 10.549 & $<0.00 I^{*}$ \\
\hline Color word reading time (CWRT, s) & $24.11 \pm 10.83$ & $18.12 \pm 7.69$ & 5.218 & $<0.001 *$ \\
\hline Word color naming time (WCRT, s) & $50.07 \pm 18.50$ & $30.08 \pm 9.80$ & 11.096 & $<0.00 I^{*}$ \\
\hline $\begin{array}{l}\text { PANSS } \\
\text { Positive symptoms }\end{array}$ & $18.85 \pm 7.03$ & - & & \\
\hline Negative symptoms & $22.36 \pm 9.00$ & - & & \\
\hline General symptoms & $39.15 \pm 18.61$ & - & & \\
\hline Total scores & $80.36 \pm 31.84$ & - & & \\
\hline
\end{tabular}

Notes: I. Systolic blood pressure, 2. Diastolic blood pressure, 3. VEGF, *:P $<0.05$.

observed in healthy controls. It indirectly reflects the lower level of VEGF in the brain of schizophrenia patients. Evidence suggests that VEGF can protect against brain cell loss, blood-brain-barrier dysfunction, spatial memory impairment, and cognitive decline in response to injury and Alzheimer disease. ${ }^{31,32}$ VEGF may also affect an improvement of hippocampal function and prefrontal cortex volume in schizophrenia. ${ }^{33,34}$ Normal levels of VEGF plays an important role in the unbalanced development of synapses in the brain through the inflammatory process. ${ }^{11}$ In our study, VEGF was inferred to have some protective affects against degeneration of the RNFL in the right eyes 
Table 2 Differences in RNFL Thickness Between Individuals with Schizophrenia and Healthy Controls

\begin{tabular}{|c|c|c|c|c|c|c|}
\hline Mean \pm SD & Schizophrenia Group & Control Group & $t$ & $p$ & $\boldsymbol{F}$ & $p^{\prime}$ \\
\hline \multicolumn{7}{|l|}{ Right RNFL } \\
\hline Total thickness $(\mu \mathrm{m})$ & $84.00 \pm 21.27$ & $101.96 \pm 13.47$ & -5.333 & $<0.001$ & 10.872 & $0.00 I^{*}$ \\
\hline Superior thickness $(\mu \mathrm{m})$ & $99.51 \pm 44.45$ & $121.70 \pm 27.45$ & -4.170 & $<0.001$ & 7.530 & $0.007^{*}$ \\
\hline Inferior thickness $(\mu \mathrm{m})$ & $105.25 \pm 44.13$ & $127.85 \pm 24.30$ & -4.461 & $<0.001$ & 6.689 & $0.011^{*}$ \\
\hline Disc area $\left(\mathrm{mm}^{2}\right)$ & $2.20 \pm 1.35$ & $2.32 \pm 0.51$ & -0.803 & 0.423 & 0.048 & 0.995 \\
\hline Cup volume $\left(\mathrm{mm}^{3}\right)$ & $0.12 \pm 0.14$ & $0.12 \pm 0.13$ & 0.267 & 0.790 & 0.561 & 0.455 \\
\hline \multicolumn{7}{|l|}{ Left RNFL $(\mu \mathrm{m})$} \\
\hline Total thickness $(\mu \mathrm{m})$ & $93.64 \pm 13.69$ & $105.36 \pm 10.81$ & -6.113 & $<0.001$ & 33.130 & $<0.00 I^{*}$ \\
\hline Superior thickness $(\mu \mathrm{m})$ & $117.84 \pm 29.03$ & $131.80 \pm 19.87$ & -3.784 & $<0.001$ & 28.546 & $<0.00 I^{*}$ \\
\hline Inferior thickness $(\mu \mathrm{m})$ & $120.84 \pm 23.64$ & $135.50 \pm 16.44$ & -4.851 & $<0.001$ & 9.838 & $0.002^{*}$ \\
\hline Disc area $\left(\mathrm{mm}^{2}\right)$ & $2.13 \pm 0.91$ & $2.26 \pm 0.43$ & -1.295 & 0.197 & 0.099 & 0.753 \\
\hline Cup volume $\left(\mathrm{mm}^{3}\right)$ & $0.17 \pm 0.15$ & $0.12 \pm 0.12$ & 2.026 & 0.044 & 0.633 & 0.427 \\
\hline \multicolumn{7}{|l|}{ Right macula $(\mu \mathrm{m})$} \\
\hline Average thickness & $257.40 \pm 39.23$ & $263.44 \pm 36.88$ & -1.244 & 0.215 & 0.716 & 0.475 \\
\hline Central thickness & $220.53 \pm 39.34$ & $222.98 \pm 20.31$ & -0.592 & 0.555 & 1.184 & 0.238 \\
\hline Inner superior & $284.27 \pm 45.56$ & $302.85 \pm 18.73$ & -0.401 & $<0.001$ & 4.755 & $<0.00 I^{*}$ \\
\hline Inner inferior & $274.23 \pm 47.36$ & $290.68 \pm 20.75$ & -3.388 & 0.001 & 3.524 & $0.00 I^{*}$ \\
\hline Inner nasal & $286.79 \pm 46.46$ & $298.22 \pm 26.10$ & -2.428 & 0.016 & 1.847 & 0.066 \\
\hline Inner temporal & $268.35 \pm 42.93$ & $282.10 \pm 19.17$ & -3.338 & 0.001 & 3.378 & $0.00 I^{*}$ \\
\hline Outer ring superior & $254.95 \pm 40.25$ & $266.67 \pm 20.44$ & -2.778 & 0.006 & 3.542 & $<0.00 I^{*}$ \\
\hline Outer inferior & $245.72 \pm 40.98$ & $256.02 \pm 19.62$ & -2.421 & 0.017 & 2.776 & $0.006 *$ \\
\hline Outer nasal & $267.80 \pm 42.71$ & $278.16 \pm 26.23$ & -2.331 & 0.021 & 2.615 & $0.010^{*}$ \\
\hline Outer temporal & $241.59 \pm 38.51$ & $247.07 \pm 21.43$ & -1.409 & 0.160 & 2.573 & $0.011^{*}$ \\
\hline \multicolumn{7}{|l|}{ Left macula $(\mu \mathrm{m})$} \\
\hline Average thickness & $254.35 \pm 39.50$ & $262.86 \pm 47.64$ & -1.499 & 0.135 & 0.614 & 0.540 \\
\hline Central thickness & $219.54 \pm 38.41$ & $228.44 \pm 25.56$ & -2.154 & 0.032 & 1.223 & 0.222 \\
\hline Inner superior & $280.48 \pm 44.58$ & $303.05 \pm 16.78$ & -5.003 & $<0.001$ & 3.701 & $<0.00 I^{*}$ \\
\hline Inner inferior & $273.43 \pm 44.14$ & $292.46 \pm 22.35$ & -4.091 & $<0.001$ & 2.989 & $0.003^{*}$ \\
\hline Inner nasal & $281.17 \pm 48.03$ & $296.80 \pm 40.59$ & -2.754 & 0.006 & 1.933 & 0.055 \\
\hline Inner temporal & $269.50 \pm 44.58$ & $287.66 \pm 16.78$ & -4.027 & $<0.001$ & 2.915 & $0.004^{*}$ \\
\hline Outer ring superior & $252.28 \pm 40.14$ & $267.93 \pm 16.99$ & -3.803 & $<0.001$ & 2.517 & $0.013^{*}$ \\
\hline Outer inferior & $242.29 \pm 39.12$ & $256.43 \pm 16.45$ & -3.529 & 0.001 & 1.606 & 0.110 \\
\hline Outer nasal & $266.74 \pm 44.96$ & $284.74 \pm 17.62$ & -3.942 & $<0.001$ & 1.879 & 0.062 \\
\hline Outer temporal & $238.50 \pm 39.11$ & $249.81 \pm 15.75$ & -2.842 & 0.005 & 1.182 & 0.239 \\
\hline
\end{tabular}

Notes: $p$ : for $t$-test; $p$ ': adjusted $p$ for analysis of covariance after controlling for age, sex, and smoking. *:P<0.05. 


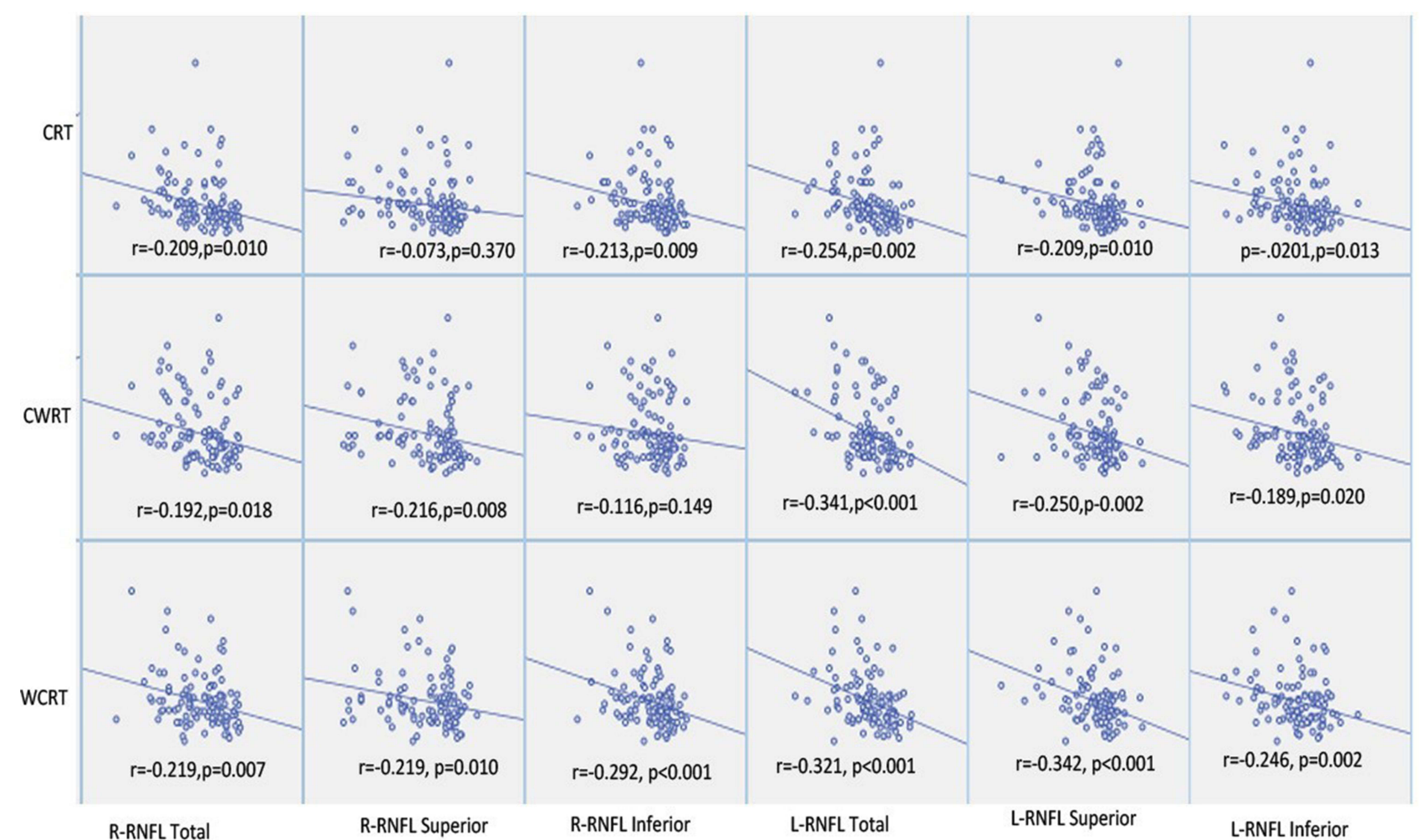

Figure 2 The relationship between the thickness of retinal nerve fiber layer (RNFL) and the scores of the Stroop test.

Abbreviations: R-RNFL, right RNFL; L-RNFL, left RNFL; CRT, color reading time; CWRT, color word reading time; WCRT, word-color naming time.

of schizophrenia patients. The effect of VEGF on the RNFL of right eyes is more prominent, which might indirectly reflect more pronounced protective effects on the left cerebral nerve circuit, according to the visual neural transmission pathway. VEGF might also provide multiform diphase regulation in response to impairment of this nerve pathway.Although cross-sectional studies affect the accuracy of speculation, the precise mechanism by which VEGF is related to cognitive impairment and RNFL change in patients with schizophrenia remains to be elucidated.

Various studies have tried to investigate visual perception as a new endophenotype of schizophrenia. ${ }^{35,36} \mathrm{SCWT}$, which was designed as a cognitive test assessed via vision, has been widely used to evaluate reading speed, selective attention, and cognitive flexibility. ${ }^{37}$ Previous research has shown that time spent in the SCWT is related to visual search and perceptual speed factors. ${ }^{38}$ Increased SCWT response time is a common feature of the impaired cognitive function observed in schizophrenia. Accordingly, in the current study, patients with schizophrenia took more time to complete the SCWT, when compared with healthy controls, which is consistent with the results reported by previous studies. 39,40
Increased SCWT response time as a measure of cognition impairment exhibited a significant relationship with the RNFL thickness in our study. This is consistent with results presented in a previous study, which found that RNFL impairment is related to visuospatial cognitive impairment. ${ }^{18}$ The retina is the first structure to receive and process environmental visual information. Some studies infer that visual deficits in schizophrenia patients are related to the parvocellular pathway or are related to the interaction between the magnocellular and parvocellular pathways containing spatial and temporal information processed. ${ }^{41,42}$ Both the two pathways originate from different types of retinal ganglion cells (RGCs). RGCs form the retinal nerve that projects for the most part to the visual cortex to generate vision. ${ }^{43}$ The association between early visual processing in the retina and cognitive performance in schizophrenia may be close. ${ }^{42}$ Changes in the senior visual nerve of the brain may be related to problems with incoming primary visual processing. Therefore, we speculate that, based on the thinner RNFL thickness observed in patients with schizophrenia, the degeneration of the retinal nerve may be associated with visual cognitive impairment. Retinal nerve cell loss reflects the neural 

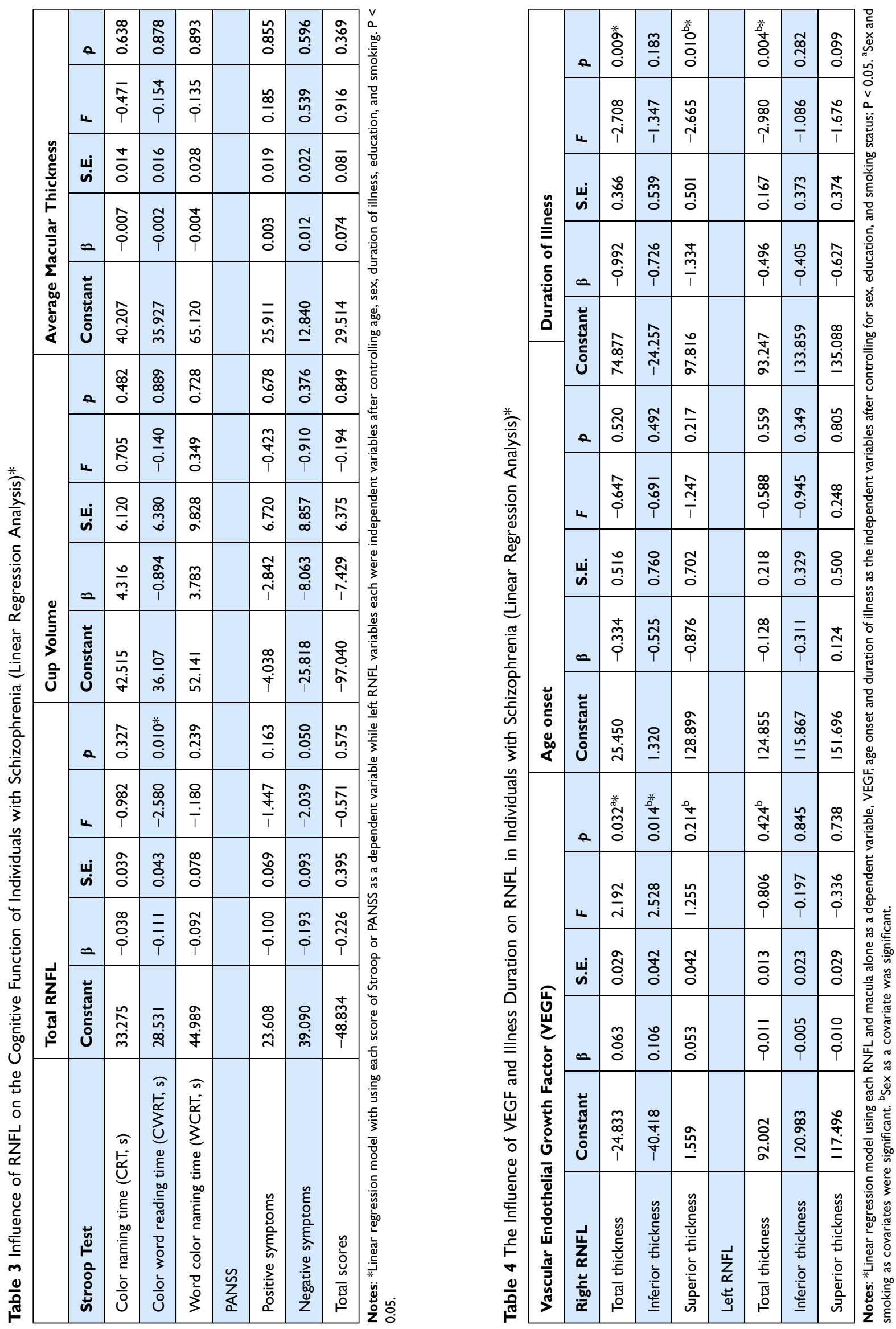
network problems in the development of the brain in schizophrenic patients, and thus the cognitive function with visual participation may be steeply impaired. Abnormal reduction of RNFL indirectly reflects the atypical development of brain structure. ${ }^{4,25}$ Further, retinal layer abnormalities might be used as a potential biomarker in schizophrenia, due to significant correlations with clinical features and visual function. ${ }^{23}$ Whether this change in the retina follows or plays a decisive role in the impairment of the cognitive development remains to be clarified.

\section{Limitations}

There are several limitations to consider in the current single-center, cross-sectional study. First, the thickness of the macula obtained with Topcon SD-OCT2002 was not a single-layer ganglion cell thickness; rather it was a cumulative ganglion cell complex owing to the automatic measurement limitation. Second, doses of antipsychotics used by patients in the current study could be a confounding factor, as it is unknown whether these medications affect the RNFL thickness. ${ }^{2}$ Third, the RNFL thickness is just one of the factors related to visual acuity. It is possible there could be other eye conditions that were not balanced in the patients. Fourth, healthy controls are taken from the staff of the hospital, this may bias the exclusion diagnosis of axis 1 due to missing anonymity. Additionally, the cross-sectional design of our study seemed to be insufficient speculation about progressive degeneration of RNFL during the course of schizophrenia. Finally, the lack of a control neuroimaging method, such as magnetic resonance imaging, makes it difficult to confirm conclusions from our results. ${ }^{44}$

\section{Conclusions}

Patients with schizophrenia exhibit thinner RNFL thickness. The decreased thickness of the RNFL may be related to impairment of cognitive tasks requiring visual participation. Cognitive impairment in tasks requiring visual involvement may be closely related to the cell loss of retinal nerve fibers. RNFL degeneration might be a potential biomarker of schizophrenia. The abnormal level of VEGF that might be involved in this degeneration of RNFL through the vascular neuroimmunology process.

\section{Acknowledgments}

We thank Professor of Ophthalmology Lvzhen Huang and her Peking University team. We would also like to thank Fude Yang and the team of our Integration of Traditional and Western Medicine Institute. This research is supported by the Beijing Municipal Science \& Technology Commission no. Z181100001718115 and the Capital's Funds for Health Improvement and Research no. 2020$1-2131$.

\section{Disclosure}

The authors report no conflicts of interest in this work.

\section{References}

1. Csernansky JG. Neurodegeneration in schizophrenia: evidence from in vivo neuroimaging studies. ScientificWorldJournal. 2007;7:135-143. doi:10.1100/tsw.2007.47

2. Silverstein SM, Rosen R. Schizophrenia and the eye. Schizophr Res Cogn. 2015;2(2):46-55. doi:10.1016/j.scog.2015.03.004

3. Cettomai D, Pulicken M, Gordon-Lipkin E, et al. Reproducibility of optical coherence tomography in multiple sclerosis. Arch Neurol. 2008;65(9):1218-1222. doi:10.1001/archneur.65.9.1218

4. Chu EM, Kolappan M, Barnes TR, Joyce EM, Ron MA. A window into the brain: an in vivo study of the retina in schizophrenia using optical coherence tomography. Psychiatry Res. 2012;203(1):89-94. doi:10.1016/j.pscychresns.2011.08.011

5. Ascaso FJ, Rodriguez-Jimenez R, Cabezón L, et al. Retinal nerve fiber layer and macular thickness in patients with schizophrenia: influence of recent illness episodes. Psychiatry Res. 2015;229(12):230-236. doi:10.1016/j.psychres.2015.07.028

6. Kim JS, Ishikawa H, Sung KR, et al. Retinal nerve fibre layer thickness measurement reproducibility improved with spectral domain optical coherence tomography. Br J Ophthalmol. 2009;93 (8):1057-1063. doi:10.1136/bjo.2009.157875

7. Braus DF, Weber-Fahr W, Tost H, Ruf M, Henn FA. Sensory information processing in neuroleptic-naive first-episode schizophrenic patients: a functional magnetic resonance imaging study. Arch Gen Psychiatry. 2002;59(8):696-701. doi:10.1001/archpsyc.59.8.696

8. Ardekani BA, Nierenberg J, Hoptman MJ, Javitt DC, Lim KO. MRI study of white matter diffusion anisotropy in schizophrenia. Neuroreport. 2003;14(16):2025-2029. doi:10.1097/01.wnr.0000093 $290.85057 .0 \mathrm{~d}$

9. van de Ven V, Rotarska JA, Oertel-Knöchel V, Dej L. Reduced intrinsic visual cortical connectivity is associated with impaired perceptual closure in schizophrenia. Neuroimage Clin. 2017;15:45-52. doi:10.1016/j.nicl.2017.04.012

10. Schaeffer DJ, Rodrigue AL, Burton CR, et al. White matter fiber integrity of the saccadic eye movement network differs between schizophrenia and healthy groups. Psychophysiology. 2017;54 (12):1967-1977. doi:10.1111/psyp.12969

11. Adams SA, Nasrallah HA. Multiple retinal anomalies in schizophrenia. Schizophr Res. 2018;195:3-12. doi:10.1016/j. schres.2017.07.018

12. Misiak B, Stramecki F, Stańczykiewicz B, Frydecka D, Lubeiro A. Vascular endothelial growth factor in patients with schizophrenia: a systematic review and meta-analysis. Prog Neuropsychopharmacol Biol Psychiatry. 2018;86:24-29. doi:10.1016/j.pnpbp.2018.05.005

13. Quaggin SE. Turning a blind eye to anti-VEGF toxicities. $J$ Clin Invest. 2012;122(11):3849-3851. doi:10.1172/JCI65509

14. Fehr T, Wiedenmann P, Herrmann M. Nicotine Stroop and addiction memory-an ERP study. Int J Psychophysiol. 2006;62(2):224-232. doi:10.1016/j.ijpsycho.2006.01.011

15. Spreen O, Strauss E. A Compendium of Neuropsychological Tests: Administration, Norms and Commentary. 2nd ed. New York: Oxford University Press; 1998. 
16. Jonas JB, Wang N, Wang S, et al. Retinal vessel diameter and estimated cerebrospinal fluid pressure in arterial hypertension: the Beijing Eye Study. Am J Hypertens. 2014;27(9):1170-1178. doi:10.1093/ajh/hpu037

17. Meier MH, Gillespie NA, Hansell NK, et al. Retinal microvessels reflect familial vulnerability to psychotic symptoms: a comparison of twins discordant for psychotic symptoms and controls. Schizophr Res. 2015;164(1-3):47-52. doi:10.1016/j.schres.2015.01.045

18. Liu Y, Huang L, Tong Y, Chen J, Gao D, Yang F. Association of retinal nerve fiber abnormalities with serum CNTF and cognitive functions in schizophrenia patients. PeerJ. 2020;8:e9279. doi: $10.7717 /$ peerj.9279

19. Chen K, Huang L, Lin B, Zhou Y, Zhao Q, Guo Q. The Number of Items on Each Stroop Test Card Is Unrelated to Its Sensitivity. Neuropsychobiology. 2019;77(1):38-44. doi:10.1159/000493553

20. Periáñez JA, Lubrini G, García-Gutiérrez A, Ríos-Lago M. Construct Validity of the Stroop Color-Word Test: influence of Speed of Visual Search, Verbal Fluency, Working Memory, Cognitive Flexibility, and Conflict Monitoring. Arch Clin Neuropsychol. 2020. doi:10.1093/ arclin/acaa034

21. Kay SR, Fiszbein A, Opler LA. The positive and negative syndrome scale (PANSS) for schizophrenia. Schizophr Bull. 1987;13 (2):261-276. doi:10.1093/schbul/13.2.261

22. Pan J, Zhou Y, Xiang Y, Yu J. Retinal nerve fiber layer thickness changes in Schizophrenia: a meta-analysis of case-control studies. Psychiatry Res. 2018;270:786-791. doi:10.1016/j.psychres.2018.10.075

23. Samani NN, Proudlock FA, Siram V, et al. Retinal Layer Abnormalities as Biomarkers of Schizophrenia. Schizophr Bull. 2018;44(4):876-885. doi:10.1093/schbul/sbx130

24. Silverstein SM, Paterno D, Cherneski L, Green S. Optical coherence tomography indices of structural retinal pathology in schizophrenia. Psychol Med. 2018;48(12):2023-2033. doi:10.1017/S00332917 17003555

25. Lee WW, Tajunisah I, Sharmilla K, Peyman M, Subrayan V. Retinal nerve fiber layer structure abnormalities in schizophrenia and its relationship to disease state: evidence from optical coherence tomography. Invest Ophthalmol Vis Sci. 2013;54(12):7785-7792. doi:10.1167/iovs.13-12534

26. Kazakos CT, Karageorgiou V. Retinal Changes in Schizophrenia: a Systematic Review and Meta-analysis Based on Individual Participant Data. Schizophr Bull. 2020;46(1):27-42. doi:10.1093/ schbul/sbz106

27. Fatemi SH, Folsom TD. The neurodevelopmental hypothesis of schizophrenia, revisited. Schizophr Bull. 2009;35(3):528-548. doi:10.1093/schbul/sbn 187

28. Roberts GW. Schizophrenia: a neuropathological perspective. $\mathrm{Br}$ J Psychiatry. 1991;158:8-17. doi:10.1192/bjp.158.1.8

29. Hashimoto N, Ito YM, Okada N, et al. The effect of duration of illness and antipsychotics on subcortical volumes in schizophrenia: analysis of 778 subjects. Neuroimage Clin. 2018;17:563-569. doi:10.1016/j.nicl.2017.11.004

30. Husain N, Awasthi S, Haris M, Gupta RK, Husain M. Vascular endothelial growth factor as a marker of disease activity in neurotuberculosis. $J$ Infect. 2008;56(2):114-119. doi:10.1016/j. jinf.2007.11.004
31. Hohman TJ, Bell SP, Jefferson AL. The role of vascular endothelial growth factor in neurodegeneration and cognitive decline: exploring interactions with biomarkers of Alzheimer disease. JAMA Neurol. 2015;72(5):520-529. doi:10.1001/jamaneurol.2014.4761

32. Taylor SL, Trudeau D, Arnold B, et al. VEGF can protect against blood brain barrier dysfunction, dendritic spine loss and spatial memory impairment in an experimental model of diabetes. Neurobiol Dis. 2015;78:1-11. doi:10.1016/j.nbd.2015.03.022

33. During MJ, Cao L. VEGF, a mediator of the effect of experience on hippocampal neurogenesis. Curr Alzheimer Res. 2006;3(1):29-33. doi:10.2174/156720506775697133

34. Pillai A, Howell KR, Ahmed AO, et al. Association of serum VEGF levels with prefrontal cortex volume in schizophrenia. Mol Psychiatry. 2016;21(5):686-692. doi:10.1038/mp.2015.96

35. Gottesman II, Gould TD. The endophenotype concept in psychiatry: etymology and strategic intentions. Am J Psychiatry. 2003;160 (4):636-645. doi:10.1176/appi.ajp.160.4.636

36. Gracitelli CP, Abe RY, Diniz-Filho A, Vaz-de-lima FB, Paranhos A, Medeiros FA. Ophthalmology issues in schizophrenia. Curr Psychiatry Rep. 2015;17(5):28. doi:10.1007/s11920-015-0569-x

37. Brugnolo A, De Carli F, Accardo J, et al. An updated Italian normative dataset for the Stroop color word test (SCWT). Neurol Sci. 2016;37(3):365-372. doi:10.1007/s10072-015-2428-2

38. Sánchez-Cubillo I, Periáñez JA, Adrover-Roig D, et al. Construct validity of the Trail Making Test: role of task-switching, working memory, inhibition/interference control, and visuomotor abilities. $J$ Int Neuropsychol Soc. 2009;15(3):438-450. doi:10.1017/ S1355617709090626

39. Hagh-Shenas H, Toobai S, Makaremi A. Selective, sustained, and shift in attention in patients with diagnoses of schizophrenia. Percept Mot Skills. 2002;95(3 Pt 2):1087-1095. doi:10.2466/ pms.2002.95.3f.1087

40. Westerhausen R, Kompus K, Hugdahl K. Impaired cognitive inhibition in schizophrenia: a meta-analysis of the Stroop interference effect. Schizophr Res. 2011;133(1-3):172-181. doi:10.1016/j. schres.2011.08.025

41. Delord S, Ducato MG, Pins D, et al. Psychophysical assessment of magno- and parvocellular function in schizophrenia. Vis Neurosci. 2006;23(3-4):645-650. doi:10.1017/S0952523806233017

42. Hever F, Sahin D, Aschenbrenner S, et al. Visual N80 latency as a marker of neuropsychological performance in schizophrenia: evidence for bottom-up cognitive models. Clin Neurophysiol. 2021;132 (4):872-885. doi:10.1016/j.clinph.2021.01.007

43. Gagné AM, Hébert M, Maziade M. Revisiting visual dysfunctions in schizophrenia from the retina to the cortical cells: a manifestation of defective neurodevelopment. Prog Neuropsychopharmacol Biol Psychiatry. 2015;62:29-34. doi:10.1016/j.pnpbp.2015.04.007

44. Kalenderoglu A, Çelik M, Sevgi-Karadag A, Egilmez OB. Optic coherence tomography shows inflammation and degeneration in major depressive disorder patients correlated with disease severity. J Affect Disord. 2016;204:159-165. doi:10.1016/j.jad.2016.06.039
Neuropsychiatric Disease and Treatment

\section{Publish your work in this journal}

Neuropsychiatric Disease and Treatment is an international, peerreviewed journal of clinical therapeutics and pharmacology focusing on concise rapid reporting of clinical or pre-clinical studies on a range of neuropsychiatric and neurological disorders. This journal is indexed on PubMed Central, the 'PsycINFO' database and CAS, and is the official journal of The International Neuropsychiatric Association (INA). The manuscript management system is completely online and includes a very quick and fair peer-review system, which is all easy to use. Visit http://www.dovepress.com/testimonials.php to read real quotes from published authors. 